\title{
Prospective evaluation of aldosterone LC-MS/ MS-specific cutoffs for the saline infusion test
}

\author{
Charlotte Michaela Fries 1,*, Yoon Ju Bae 2,*, Nada Rayes ${ }^{3}$, Benjamin Sandner', Berend Isermann², \\ Michael Stumvoll' ${ }^{1}$, Valentina Fagotto ${ }^{4}$, Martin Reincke ${ }^{4}$, Martin Bidlingmaier ${ }^{4}$, Vogel Mandy ${ }^{5}$, Jürgen Kratzsch² \\ and Wiebke Kristin Fenske ${ }^{1,6}$
}

\begin{abstract}
'Medical Department III, Endocrinology, Nephrology, Rheumatology, University Hospital of Leipzig, Leipzig, Germany, 2Institute of Laboratory Medicine, Clinical Chemistry, and Molecular Diagnostics, University of Leipzig, Leipzig, Germany, ${ }^{3}$ Endocrine Surgery, Department for Visceral, Transplant, Thoracic and Vascular Surgery, University Hospital of Leipzig, Leipzig, Germany, ${ }^{4}$ Department of Medicine IV, Klinikum der Universität, Ludwig-MaximiliansUniversität, München, Germany, ${ }^{5}$ Leipzig Research Center for Civilization Diseases (LIFE Child), University of Leipzig, Leipzig, Germany, and ${ }^{6}$ Integrated Research and Treatment Centre for Adiposity Diseases, Department of Medicine, University of Leipzig, Leipzig, Germany
\end{abstract}

*(C M Fries and Y J Bae contributed equally to this work)

Correspondence should be addressed to C M Fries or W K Fenske Email charlotte.fries@medizin. uni-leipzig.de or wiebkekristin.fenske@ medizin.uni-leipzig.de

\begin{abstract}
Objective: Liquid chromatography coupled to tandem mass spectrometry (LC-MS/MS) has become state of the art for the quantitative analysis of steroid hormones. Although method comparisons show that aldosterone measurement using LC-MS/MS yields considerably lower levels than immunoassays (IAs), method-specific cutoff values for primary aldosteronism (PA) are largely missing. Objective of this study was to analyze the diagnostic accuracy of proposed LC-MS/MS-specific cutoff values for the saline infusion test (SIT).

Design and methods: From 2016 to 2019, 104 consecutive patients suspected of PA underwent the SIT and captopril challenge test in the tertiary medical center at the University Hospital of Leipzig, Germany. Patients with positive case confirmation underwent adrenal imaging and adrenal venous sampling for subtype classification.

Results: Overall, proposed assay-specific PAC $\mathrm{LC}_{\mathrm{LS} / \mathrm{MS}}$ cutoff values for the SIT achieved higher diagnostic accuracy than established $\mathrm{PAC}_{\mathrm{IA}}$ values with a sensitivity and specificity of $87.5 \%$ (95\% Cl: $\left.71.0-96.5\right)$ and $97 \%$ (95\% Cl: 89.6-99.6) for a cutoff of $120 \mathrm{pmol} / \mathrm{L}$ and $93.8 \%$ (95\% Cl: 79.2-99.2) and $92.5 \%$ (95\% Cl: 83.4-97.5) for a cutoff of $94 \mathrm{pmol} / \mathrm{L}$. The most accurate post-SIT PAC LC-MS/MS Cutoff value in this study was $83 \mathrm{pmol} / \mathrm{L}$, yielding a sensitivity and specificity of $96.9 \%$ (95\% Cl: 83.8-99.9) and 92.5\% (95\% Cl: 83.4-97.5), respectively.

Conclusions: The present data confirm the need for the implication of lower method-specific aldosterone cutoff values for the diagnosis of PA with LC-MS/MS based aldosterone measurement.
\end{abstract}

\section{Introduction}

Primary aldosteronism (PA) is the most common curable cause of secondary hypertension and accounts for approximately $>5 \%$ of hypertension cases in general and $>10 \%$ in speciality settings $(1,2,3)$. Patients with PA have higher cardiovascular morbidity and mortality than age- and sex-matched patients suffering from essential arterial hypertension (EAH) with similar blood pressure levels (4). Early diagnosis and causal treatment (like unilateral adrenalectomy or specific drugs antagonizing aldosterone action in uni- or bilateral PA, respectively) are critical to prevent cardiovascular complications and end organ damage (5). The aldosterone/renin-ratio (ARR) is the primary screening test for PA, which is calculated from measurement of plasma aldosterone concentration

Published by Bioscientifica Ltd. 
(PAC) and either the concentration or activity of renin in a morning blood sample obtained from a seated patient. Due to the high false-positive rate of ARR values and numerous influencing factors $(4,6,7,8)$, confirmatory testing is of particular importance for diagnosis of PA. Oral sodium loading, saline infusion, captopril challenge and fludrocortisone suppression are the most commonly used tests for the confirmation of PA with varying reported diagnostic accuracy and reliability $(4,9,10,11,12)$. Which of these confirmatory tests should be used in the diagnosis of PA is still up for debate as there is insufficient evidence to support the choice of one optimal test. The Endocrine Society (ES) Clinical Practice Guidelines therefore recommends no 'gold standard' confirmatory test, but the use of one or more tests according to center preferences and local expertise (4). The saline infusion test (SIT) is often chosen in clinical practice because it is easy and safe to perform, has low costs and allows outpatient testing $(13,14,15,16)$. Although the SIT is mostly performed in a recumbent position (4), a recent study could show higher sensitivity while maintaining similar specificity when performing the SIT in a seated position, presumably due to responsiveness of aldosterone to an upright position (17).

All diagnostic tests in PA require accurate, precise, and standardized measurements of plasma or serum aldosterone. Unfortunately, however, clinical analysis of aldosterone is technically challenging given its relatively low concentration in plasma at picomolar concentrations and the inherent difficulty in discrimination from aldosterone metabolites (18). Moreover, the different commercially available analytical methods for aldosterone and renin measurement constitute a major challenge to the diagnostic workup of PA (19). Especially, the most commonly used immunoassays (IAs) have been criticized for showing high cross-reactivity at low PACs and a pronounced inter-method variability, thus essentially limiting their uncritical application for confirmatory testing $(20,21,22,23)$. To overcome these limitations, clinical laboratories turned to LC-MS/MS for analysis of aldosterone and other steroids, an analytical method that does not demonstrate cross-reactivity but an improved diagnostic accuracy compared to traditional IAs $(24,25$, $26,27)$. However, LC-MS/MS yields significantly lower values of PAC compared to IAs $(22,25,26,28,29,30$, $31)$, which highlights the need for establishing assayspecific cutoffs for PA screening and confirmatory testing (32). We recently reported about the poor correlation and variable outcome of PAC measured within the German Conn's registry during the recumbent SIT by different IA and LC-MS/MS methods (23). These data indicate that reduction in current diagnostic threshold values and the establishment of method-specific cutoffs for screening and confirmatory testing of PA is necessary when switching to LC-MS/MS in clinical routine. The primary aim of the current study was to evaluate the diagnostic performance of two different $\mathrm{PAC}_{\mathrm{LC}-\mathrm{MS} / \mathrm{Ms}}$-specific threshold values for the SIT confirmatory test, which were derived from two different analytical approaches in an independent population of patients suspected of PA who had undergone SIT in our center.

\section{Subjects and methods}

\section{Study design and participants}

This study was conducted between February 2016 and March 2019 in the tertiary medical center at the University Hospital of Leipzig, Germany. It was approved by the Ethical Committee of the University of Leipzig (117/19-ek).

Hypertensive patients who were consecutively referred to our hospital for confirmatory testing were subjected to the recumbent SIT and to the captopril challenge test (CCT) to confirm or exclude PA as the cause of their hypertension (Table 1). Referral was based on outpatient ARR screening values according to the individual laboratories effective cutoff values. Functional testing was performed on an inpatient basis. Clinical characteristics of subjects are shown in Table 2 .

\section{Test protocols}

For the SIT and CCT, mineralocorticoid receptor antagonists and potassium-sparing diuretics were discontinued 4 weeks prior to testing. Other interfering drugs such as angiotensin-converting enzyme inhibitors, angiotensin receptor blockers, renin inhibitors and $\beta$-blockers were withdrawn for 2 weeks prior to testing. If testing under interfering drugs due to incompliance or severely uncontrolled hypertension led to inconclusive diagnostic results, testing was repeated or the subject excluded from data analysis. Long-acting calcium channel blockers (e.g. verapamil), $\alpha-1$ receptor antagonists (e.g. doxazosin) or vasodilatators (dihydralazine) were prescribed for bridging.

For the SIT, 2 L of $0.9 \%$ saline solution were administered intravenously over a period of $4 \mathrm{~h}$ while patients remained in a recumbent position. The CCT was 
Table 1 Criteria to confirm or exclude PA. Patients with biochemical and clinical picture of PA without conclusive evidence to establish the diagnosis of APA were presumed to have IHA.

Criteria to confirm PA

1. Screening criteria according the ES Clinical Practice Guidelines (4) and

2. Biochemical diagnosis of PA consisting of Positive ARR (according to individual assays used) and Spontaneous $\mathrm{PAC}>550 \mathrm{pmol} / \mathrm{L}$ and spontaneous hypokalemia and suppressed renin or Positive confirmatory testing and

3. Cure or improvement of clinical hypertension (reduced blood pressure with reduced antihypertensive medication) and/or correction of biochemical picture (normal potassium, unsuppressed renin, negative ARR) exclusively in response to mineralocorticoid receptor antagonists or adrenalectomy

Additional criteria to establish APA

1. Factor 3 lateralized aldosterone secretion in AVS

2. Presence of an adrenal mass on CT and/or MRI and/or surgery

3. No increase in postural stimulation test if performed

APA, aldosterone-producing adenoma; ARR, aldosterone/renin-ratio; AVS, adrenal venous sampling; ES, Endocrine Society; IHA, idiopathic aldosteronism; PA, primary aldosteronism; PAC, plasma aldosterone concentration.

performed with $25 \mathrm{mg}$ of captopril orally administered. Before and after each test, blood was drawn for the measurement of PAC, plasma renin concentration (PRC) and serum potassium. Sodium intake was unrestricted and potassium supplementation allowed before testing. Patients with severe uncontrolled hypertension (systolic blood pressure $>180 \mathrm{mmHg}$ or diastolic blood pressure $>100$ diastolic mmHg), NYHA III-IV, or myocardial infarction within the last 3 months prior testing were excluded from volume exposure. Failure to establish an unequivocal diagnosis with the diagnostic criteria listed subsequently led to exclusion (Fig. 1). Consent has been obtained from each patient after full explanation of the purpose and nature of all procedures used.

\section{LC-MS/MS-based quantification of PAC and measurement of PRC}

For this study, PAC was quantified using LC-MS/MS for steroid profile as we specified before (28). 6PLUS1®multilevel serum calibrators and MassCheck® steroid serum controls were purchased from Chromsystems Instruments \& Chemicals GmbH (Munich, Germany). Lower limit of quantification (LLOQ) for PAC was $83 \mathrm{pmol} / \mathrm{L}$. Intra-assay coefficient of variance $(\mathrm{CV})$ were $3.3-7.4 \%$ at concentrations of $100-2100 \mathrm{pmol} / \mathrm{L}(n=10)$ and inter-assay CV $5.2-9.7 \%$ at concentrations of 200-2100 pmol/L $(n=58)$.

The PRC was measured using chemiluminescence immunoassay technology, LIASISON® purchased from DiaSorin (Saluggia, Italy). Measurement was performed according to the product specification of the manufacturer. The functional sensitivity of the assay was $1.6 \mathrm{mU} / \mathrm{L}$. Intra-assay CV was $2.5-4.9 \%(n=20)$ for concentrations between 3.5 and $268 \mathrm{mU} / \mathrm{L}$. Mean inter-assay CVs were $9.13 \pm 2.33 \%$ and $5.94 \pm 2.21 \%(n=75)$ for concentrations of $9.13 \pm 2.33 \mathrm{mU} / \mathrm{L}$ as well as of $5.94 \pm 2.21 \mathrm{mU} / \mathrm{L}$.

\section{Derivation of $\mathrm{PAC}_{\mathrm{LC}-\mathrm{MS} / \mathrm{MS}}$-specific cutoff values to be tested}

To validate a diagnostic cutoff for postinfusion SIT

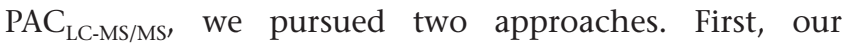

Table 2 Baseline characteristics of the study population, $n=99$. Data are expressed as mean \pm S.D. or median and IQR for non-normally distributed parameters.

\begin{tabular}{|c|c|c|c|c|}
\hline & \multirow[b]{2}{*}{ EAH } & \multicolumn{3}{|c|}{ PA } \\
\hline & & PA (total) & $\mathrm{IHA}$ & APA \\
\hline Sex (female/male) & $48 / 19$ & $14 / 18$ & $9 / 14$ & $5 / 4$ \\
\hline Age (years) & $52.0 \pm 13.4$ & $52.8 \pm 13.1$ & $55.5 \pm 11.0$ & $45.8 \pm 16.0$ \\
\hline SBP $(\mathrm{mmHg})$ & $145(134-167)$ & $168^{a}(152-179)$ & $177(160-180)$ & $155(133-174)$ \\
\hline $\mathrm{DBP}(\mathrm{mmHg})$ & $90(81-95)$ & $94(86-102)$ & $92(81-105)$ & 95 (90-99) \\
\hline $\mathrm{BMI}\left(\mathrm{kg} / \mathrm{m}^{2}\right)$ & $27.8 \pm 5.2$ & $30.1 \pm 7.1$ & $31.0 \pm 7.6$ & $27.8 \pm 4.5$ \\
\hline Serum K (mmol/L) & $4.18(4.0-4.36)$ & $3.84^{\mathrm{b}}(3.41-4.22)$ & $3.84(3.60-4.22)$ & $3.39(2.98-4.22)$ \\
\hline Prevalence of hypokalemia (\%) & 10.5 & 50.0 & 39.1 & 77.8 \\
\hline Baseline PAC (pmol/L) & $206.0(144.0-319.0)$ & $489.5^{a}(368.3-724.8)$ & $467.0(307.0-700.0)$ & $573.0(416.0-746.5)$ \\
\hline Baseline PRC (mU/L) & $5.2(2.0-8.7)$ & $1.8^{\mathrm{c}}(1.6-4.8)$ & $2.7(1.6-5.0)$ & $1.6(1.6-1.9)$ \\
\hline
\end{tabular}

${ }^{a} P<0.0001$ vs $E A H ;{ }^{b} P<0.0017$ vs $E A H ;{ }^{c} P<0.0004$ vs EAH.

APA, aldosterone-producing adenoma; DPB, diastolic blood pressure; EAH, essential hypertension; IHA, idiopathic aldosteronism; IQR, interquartile range; $\mathrm{K}$, potassium; PA, primary aldosteronism; PAC, plasma aldosterone concentration; PRC, plasma renin concentration; SBP, systolic blood pressure. 


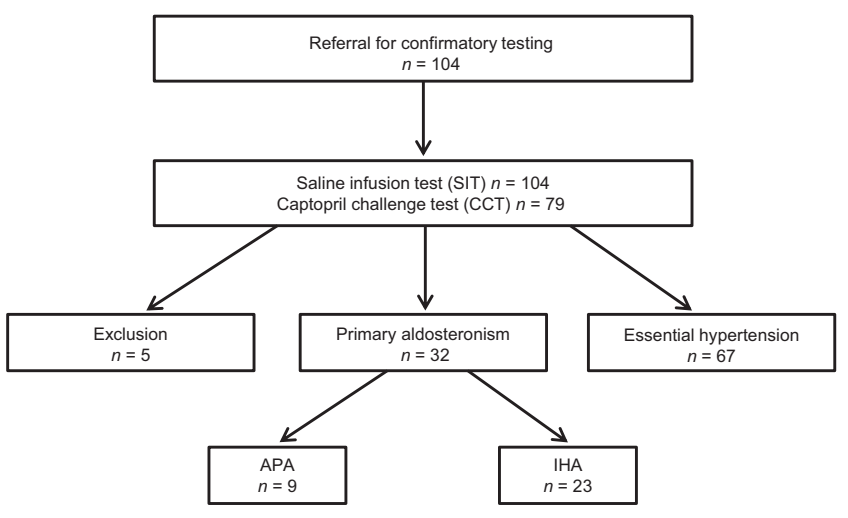

Figure 1

Flow chart displaying the diagnostic pathway from referral to diagnosis. The study cohort was comprised of 104 hypertensive patients that were referred to the University Hospital of Leipzig for confirmatory testing and underwent saline infusion test (SIT) $(n=104)$ and captopril challenge test $(n=79)$ to confirm or exclude primary aldosteronism (PA). Five patients were excluded from data analysis due to the following reasons (1.) clinical need to continue antihypertensive medication interfering with test result (two subjects), (2.) loss during diagnostic follow-up (two subjects), and (3.) technical issue resulting from suspected mislabeling of samples (one subject). APA, aldosterone-producing adenoma; IHA, idiopathic aldosteronism.

previous in-house method comparison between LC-MS/MS assay and a commercially available IA for aldosterone measurement (Aldosterone Radioimmunoassay by Beckmann Coulter, Krefeld Germany) confirmed significantly lower PACs in absolute quantification when measured by LC-MS/MS as compared to IA ( $-45 \%$ mean) (28), indicating that the adjusted cutoff should be lower than that for $\mathrm{PAC}_{\mathrm{IA}}$. Based on the previous method comparison (28), a regression equation was derived from analysis of 212 pairs of plasma samples as follows, $\mathrm{PAC}_{\mathrm{LC}-\mathrm{MS} / \mathrm{MS}}=-20.029+\left(0.732 \times \mathrm{PAC}_{\mathrm{IA}}\right) \quad$ (33). Applying this regression equation to the postinfusion SIT $\mathrm{PAC}_{\mathrm{IA}}$ cutoff of $190 \mathrm{pmol} / \mathrm{L}$, which has been found to offer the most favorable compromise between sensitivity and specificity $(13,34,35,36)$, predicted a postinfusion SIT $\mathrm{PAC}_{\mathrm{LC}-\mathrm{MS} / \mathrm{MS}}$ cutoff approximating $120 \mathrm{pmol} / \mathrm{L}$.

In a second approach, we applied the aldosterone LC-MS/MS assay to 80 samples obtained from the German Conn Registry (http://www.conn-register.de/) with confirmed $(n=39)$ and excluded PA $(n=41)$ to establish a postinfusion SIT PAC $_{\text {LC-MS/MS }}$ cutoff, which should minimize the rate of false positives for confirmatory testing. The design and methods of the German Conn
Registry have previously been described in detail $(37,38)$. In brief, the registry is a multi-center database enrolling patients with PA prospectively from 2008 onward. Using an expert endocrinologist panel classification as reference

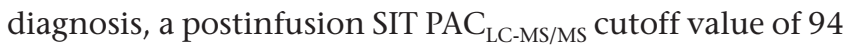
$\mathrm{pmol} / \mathrm{L}$ was found to offer the highest diagnostic accuracy to distinguish PA from EAH for the presented LC-MS/MS method (23).

\section{Diagnostic criteria}

\section{SIT and CCT}

For the SIT, a postinfusion $\mathrm{PAC}_{\mathrm{LC}-\mathrm{MS} / \mathrm{MS}} \geq 140 \mathrm{pmol} / \mathrm{L}$ (>5 ng/dL) was considered to confirm PA, while a postinfusion $\mathrm{PAC}_{\mathrm{LC}-\mathrm{MS} / \mathrm{MS}}<83 \mathrm{pmol} / \mathrm{L}(<3 \mathrm{ng} / \mathrm{dL})$ was considered to exclude PA. The reasoning for this approach was based on two notions. First, LC-MS/MS specific cutoff values should fall considerably below the IA specific cutoff, where postinfusion $\mathrm{PAC}_{\mathrm{IA}}<140 \mathrm{pmol} / \mathrm{L}$ is generally considered to make the diagnosis of PA most unlikely (4), though is still higher than the predicted LC-MS/MS cutoff values. Secondly, the fact that PAC $\mathrm{LC}_{\mathrm{LS} /}$ Ms $<83$ pmol/L marks the LLOQ for reliable PAC measurement by our LC-MS/MS assay, whereas this value is still below the predicted LC-MS/MS cutoff values. For the CCT, a suppressed $\mathrm{PAC}_{\mathrm{LC}-\mathrm{MS} / \mathrm{M} / \mathrm{S}} \geq 30 \%$ at $2 \mathrm{~h}$ after captopril allowed exclusion of PA, while a $\mathrm{PAC}_{\mathrm{LC}-\mathrm{MS} / \mathrm{M} / \mathrm{S}}$ delta $\leq 20 \%$ confirmed PA $(4,39)$.

\section{Final reference diagnosis}

In the absence of a diagnostic standard, the diagnosis to confirm or exclude PA in this study was based on clinical and biochemical criteria as specified in Table 1. Briefly, PA was confirmed in patients with positive screening criteria (4), an elevated ARR screening test (according to the individual lab assay used) in combination with a spontaneous $\mathrm{PAC}_{\mathrm{LC}-\mathrm{MS} / \mathrm{Ms}}>550 \mathrm{pmol} / \mathrm{L}(>20 \mathrm{ng} / \mathrm{dL})$, spontaneous hypokalemia, and suppressed plasma renin and/or positive confirmatory testing (Table 1). The final reference diagnosis was determined by a panel of experienced endocrinologists under careful consideration of patients' medical history, clinical presentation, screening and confirmatory test results, and exclusive response to specific treatment at follow-up. In order to establish the diagnosis aldosterone-producing adenoma (APA), lateralization in adrenal venous sampling (AVS) had to be present in combination with an identifiable mass on adrenal imaging and/or surgery (Table 1). 


\section{Subtype classification}

All patients with confirmed PA underwent adrenal imaging with high resolution CT or MRI. If surgical therapy was favored, AVS was performed (without cosyntropin stimulation) as previously described (40) to determine uni- vs bilateral aldosterone excess. Criteria for adequate catheterization in AVS were a selectivity index $\geq 3$ and for lateralization the cortisol-corrected aldosterone ratio had to be $\geq 3: 1$ (high side to low side).

\section{Statistical analysis}

Data are expressed as mean \pm S.D. or median and interquartile range (IQR) for parameters that are not normally distributed. Non-normally distributed group comparisons were performed with the Mann-Whitney $U$-test. Receiver operating characteristic (ROC) curves and Youden's Index (Sensitivity+Specificity-1) were used to analyze the performance of different cutoff values for the SIT and ARR under LC-MS/MS conditions. Diagnostic accuracy of the SIT and ARR were determined via the area under the ROC curve $\left(\mathrm{AUC}_{\mathrm{ROC}}\right)$. Statistical analyses were performed with GraphPad Prism 8.1.1 and MedCalc 19.0.5.

\section{Results}

\section{Baseline characteristics}

Of the 104 consecutive patients (64 females) referred to the University Hospital of Leipzig for confirmatory testing, 99 patients could be included (Fig. 1). Clinical baseline characteristics are summarized in Table 2. Thirtytwo patients received a final diagnosis of PA (32.3\%) and in 67 patients (67.7\%) PA could be excluded. There were no significant differences in age or BMI between the diagnostic groups. Distribution of sex was disproportionate in the EAH group with a higher percentage of female patients. Patients with PA had significantly higher systolic blood pressure and PAC at baseline compared to patients with EAH. Potassium levels and PRC were significantly lower in the PA compared to EAH group. Hypokalemia $(<3.5 \mathrm{mmol} / \mathrm{L})$ was more often present in patients diagnosed with PA than with EAH (50\% vs $10.5 \%)$.

\section{Comparison of baseline and postinfusion $\mathrm{PAC}_{\mathrm{LC}-\mathrm{MS} / \mathrm{MS}}$ levels during the SIT confirmatory test}

Median and individual $\mathrm{PAC}_{\mathrm{LC}-\mathrm{MS} / \mathrm{MSs}}$ at baseline and after saline infusion during the recumbent SIT are illustrated in Fig. 2 and Table 3, respectively. The baseline and postinfusion SIT $\mathrm{PAC}_{\mathrm{LC}-\mathrm{MS} / \mathrm{MSs}}$ were significantly higher in the PA group compared to the EAH group $(P<0.0001)$, while post-SIT $\mathrm{PAC}_{\mathrm{LC}-\mathrm{MS} / \mathrm{MSs}}$ were higher in the APA group compared to patients diagnosed with idiopathic aldosteronism (IHA) $(P<0.0149)$. We found that all but five patients from the EAH group (92.5\%) showed a suppressed $\mathrm{PAC}_{\mathrm{LC}-\mathrm{MS} / \mathrm{MS}}$ of $<83 \mathrm{pmol} / \mathrm{L}$ after SIT. This marks the LLOQ in our laboratory for reliable measurements of PAC, meaning that lower values cannot be determined with sufficient reliability. Overlap of postinfusion $\mathrm{PAC}_{\mathrm{LC}-\mathrm{MS} / \mathrm{MS}}$ values was found between the EAH and PA group, which was limited to results between patients diagnosed as EAH and IHA, respectively (Fig. 2B). A modest rise of blood pressure was seen occasionally during SIT with overall high tolerability.

\section{Diagnostic performance of selected $\mathrm{PAC}_{\mathrm{LC}-\mathrm{MS} / \mathrm{MS}}$ cutoff values for SIT confirmatory testing}

Both cohort independently derived cutoff values were tested in the present validation cohort against the background of the final reference diagnosis. The regression equation-derived cutoff value of postinfusion $\mathrm{PAC}_{\mathrm{LC}-\mathrm{MS} / \mathrm{MS}}$ of $120 \mathrm{pmol} / \mathrm{L}$ resulted in correct diagnostic classification
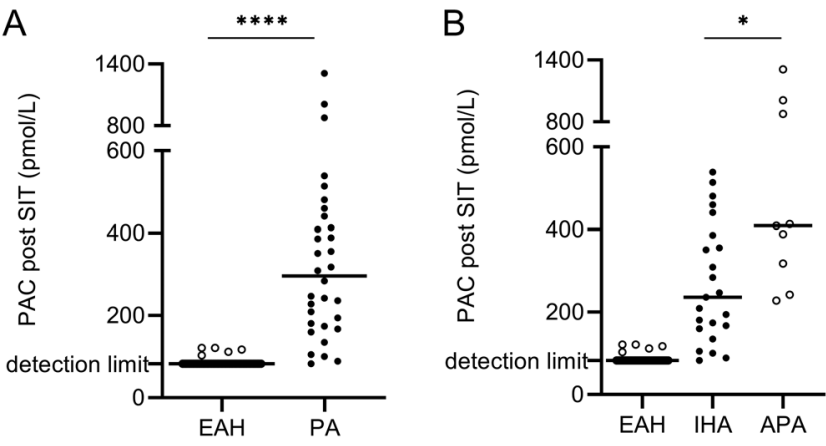

\section{Figure 2}

The diagram shows the plasma aldosterone concentrations $\left(\mathrm{PAC}_{\mathrm{LC}-\mathrm{MS} / \mathrm{MS}}\right)$ after the saline infusion test (SIT) in patients diagnosed with essential arterial hypertension (EAH) vs primary aldosteronism at large (PA) (A) and vs patients sub-classified as aldosterone-producing adenoma (APA) or idiopathic aldosteronism (IHA) (B). Post-infusion median $\mathrm{PAC}_{\mathrm{LC}-\mathrm{MS} / \mathrm{MS}}$ was significantly higher in the PA group $(P<$ $0.0001)$ compared to the EAH group and significantly higher between patients sub-classified as APA vs IHA $(P<0.0149)$. Moderate overlap occurred at low $\mathrm{PAC}_{\mathrm{LC}-\mathrm{MS} / \mathrm{MS}}$ above the lower limit of quantification (LLOQ at $83 \mathrm{pmol} / \mathrm{L}$ ) between $\mathrm{EAH}$ and IHA groups. 
Table $3 \mathrm{PAC}_{\mathrm{LC}-\mathrm{MS} / \mathrm{MS}}$ before and after saline infusion during the recumbent SIT. Data are expressed as median (IQR).

\begin{tabular}{|c|c|c|}
\hline & \multicolumn{2}{|c|}{$\mathrm{PAC}_{\mathrm{LC}-\mathrm{MS} / \mathrm{Ms}}$ in $\mathrm{pmol} / \mathrm{L}$} \\
\hline & Before SIT & After SIT \\
\hline $\mathrm{EAH}(n=67)$ & $206.0(144.0-319.0)$ & $83.0(83.0-83.0)$ \\
\hline PA (total) & $489.5^{a}(368.3-724.8)$ & $296.5^{a}(175.8-434.0)$ \\
\hline $\operatorname{IHA}(n=23)$ & 467.0 (307.0-700.0) & $236.0^{\mathrm{a}}(160.0-386.0)$ \\
\hline $\operatorname{APA}(n=9)$ & $573.0(416.0-746.5)$ & $409.0^{\mathrm{b}}(280.0-943.0)$ \\
\hline
\end{tabular}

a $P<0.0001$ vs $\mathrm{EAH} ;{ }^{\mathrm{b}} P<0.0149$ vs IHA.

APA, aldosterone-producing adenoma; EAH, essential hypertension; $I H A$, idiopathic aldosteronism; IQR, interquartile range; $P A$, primary aldosteronism; PAC, plasma aldosterone concentration; SIT, saline infusion test.

in 93 of 99 patients (93.9\%). Four patients with PA were falsely classified as EAH, and two patients with EAH were misclassified as PA. By applying the Conn's Registry derived lower cutoff value of $94 \mathrm{pmol} / \mathrm{L}$ (23), consistent results with the final reference diagnoses were found in 92 patients (92.9\%), while two patients with PA were falsely classified as unaffected and five patients with EAH were falsely diagnosed with PA.

Comparing the overall diagnostic accuracy of both SIT cutoff values, the $\mathrm{PAC}_{\mathrm{LC}-\mathrm{MS} / \mathrm{MS}}$ of $120 \mathrm{pmol} / \mathrm{L}$ showed a sensitivity and specificity of $87.5 \%$ (95\% CI: 71.0-96.5) and 97\% (95\% CI: 89.6-99.6), whereas the $\mathrm{PAC}_{\mathrm{LC}-\mathrm{MS} / \mathrm{MS}}$ of $94 \mathrm{pmol} / \mathrm{L}$ achieved corresponding values of $93.8 \%$ (95\% CI: 79.2-99.2) and 92.5\% (95\% CI: 83.4-97.5).

To identify next the most beneficial cutoff value for SIT confirmatory testing in our validation cohort, we performed a ROC curve analysis (Fig. 3). Here, the maximum value of the Youden's index was reached at the LLOQ of our assay at $83 \mathrm{pmol} / \mathrm{L}$ with a sensitivity and specificity of $96.9 \%$ (95\% CI: 83.8-99.9) and $92.5 \%$ (95\% CI: 83.4-97.5) with corresponding positive and negative predictive values of $86.1 \%$ and $98.4 \%$.

The range of variable diagnostic accuracy depending on the SIT confirmatory cutoff value chosen for $\mathrm{PAC}_{\mathrm{LC}-\mathrm{MS} / \mathrm{MS}}$ measurement is illustrated in Table 4. The data indicate that the higher the chosen SIT cutoff value, the higher the diagnostic specificity and precision, which is at the expense of the sensitivity and false-negative results.

\section{Diagnostic performance of different ARR values under LC-MS/MS conditions}

We finally sought to determine the ARR value that provides the clinically most sensitive screening performance under LC-MS/MS conditions in our cohort. PAC $_{\mathrm{LC}-\mathrm{MS} / \mathrm{MS}}$ and PRC levels that were obtained during baseline SIT sampling among the 99 patients with confirmed or excluded PA

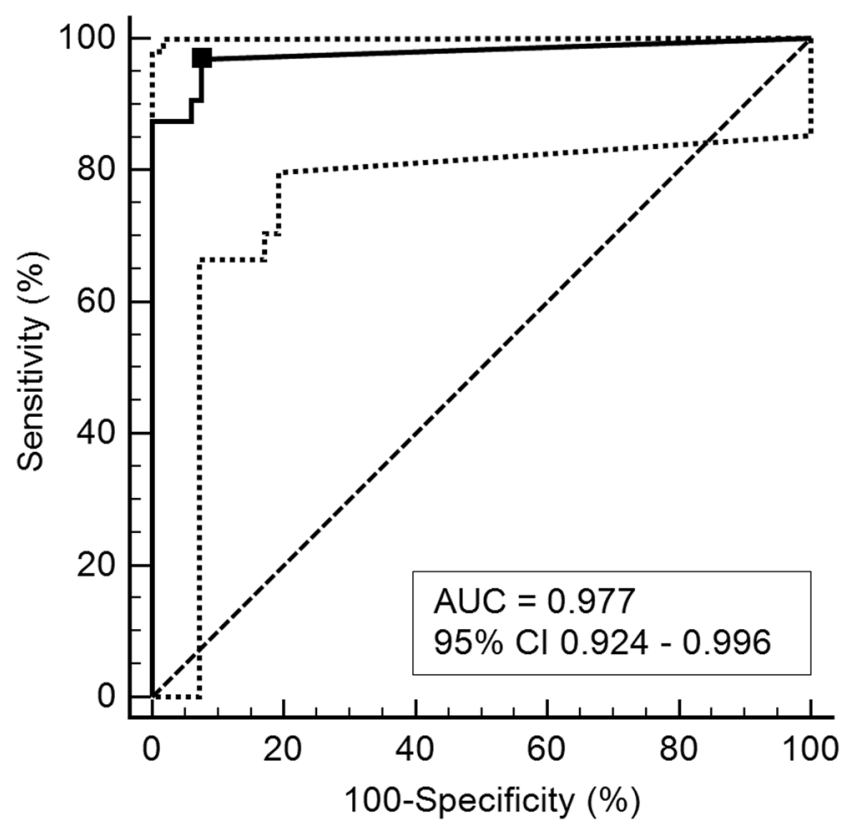

Figure 3

The receiver operating characteristics (ROC) curve for the plasma aldosterone concentration ( $P A C_{\text {LC-MS/MS }}$ ) after saline infusion for identification of primary aldosteronism (PA) is shown with an area under the ROC curve ( $A \cup C_{R O C}$ ) of 0.977 (95\% Cl: 0.9244-0.996; $P<0.001$ ). The black square marks the optimal cutoff value in our cohort at $83 \mathrm{pmol} / \mathrm{L}$ identified by Youden's index $(\mathrm{J}=$ sensitivity + specificity -1$)$. Dashed lines indicate the $95 \% \mathrm{Cl}$.

were used to calculate screening performances of ARR values (Fig. 4 and Table 5). Here, an ARR value of 53 $\mathrm{pmol} / \mathrm{mU}$ turned out as the most sensitive compromise by yielding a false-negative rate of $3.1 \%$ at the expense of a low specificity and a false-positive rate of $40.3 \%$.

\section{Discussion}

An accurate and reliable confirmatory test is a fundamental prerequisite to confirm or exclude PA and to prevent patients with falsely positive ARR screening results (7, 41) from the unnecessary risk of invasive diagnostic subclassification and/or surgical treatment. The recumbent SIT is currently still the most frequently used confirmatory test to demonstrate the absence of physiological aldosterone suppression (42). A critical and vulnerable point, however, of the SIT and other confirmatory tests that make a diagnostic decision reliant on an absolute threshold value of achieved PAC suppression (4) is the poor inter-laboratory reproducibility and limited 
Table 4 Diagnostic accuracy of different cutoff values for PAC levels after SIT under LC-MS/MS conditions.

\begin{tabular}{l}
\hline PAC $(\mathrm{pmol} / \mathrm{L})$ \\
\hline$>83$ \\
$>90$ \\
$>94$ \\
$>100$ \\
$>110$ \\
$>120$ \\
$>130$ \\
$>140$ \\
$>190$ \\
$>280$
\end{tabular}

\begin{tabular}{ccc}
\hline \multicolumn{2}{c}{ Sensitivity } \\
\hline \multicolumn{1}{c}{$\%(95 \% \mathrm{Cl})$} & & no./total no. \\
\cline { 1 - 1 } \cline { 1 - 2 } $96.9(83.8-99.9)$ & & $31 / 32$ \\
$93.8(79.2-99.2)$ & & $30 / 32$ \\
$93.8(79.2-99.2)$ & & $30 / 32$ \\
$90.6(75.0-98.0)$ & & $29 / 32$ \\
$87.5(71.0-96.5)$ & & $28 / 32$ \\
$87.5(71.0-96.5)$ & & $28 / 32$ \\
$87.5(71.0-96.5)$ & & $28 / 32$ \\
$84.4(67.2-94.7)$ & & $27 / 32$ \\
$71.9(53.3-86.3)$ & & $23 / 32$ \\
$53.1(34.7-70.9)$ & $17 / 32$ \\
\hline
\end{tabular}

\begin{tabular}{|c|c|}
\hline \multicolumn{2}{|c|}{ Specificity } \\
\hline$\%(95 \% \mathrm{Cl})$ & no./total no. \\
\hline 92.5 (83.4-97.5) & $62 / 67$ \\
\hline 92.5 (83.4-97.5) & $62 / 67$ \\
\hline 92.5 (83.4-97.5) & $62 / 67$ \\
\hline 92.5 (83.4-97.5) & $62 / 67$ \\
\hline 94.0 (85.4-98.3) & $63 / 67$ \\
\hline 97.0 (89.6-99.6) & $65 / 67$ \\
\hline $100.0(94.6-100.0)$ & $67 / 67$ \\
\hline $100.0(94.6-100.0)$ & $67 / 67$ \\
\hline $100.0(94.6-100.0)$ & $67 / 67$ \\
\hline $100.0(94.6-100.0)$ & $67 / 67$ \\
\hline
\end{tabular}

\begin{tabular}{|c|c|}
\hline \multicolumn{2}{|c|}{ PPV } \\
\hline$\%$ & no./total no. \\
\hline 86.1 & $31 / 36$ \\
\hline 85.7 & $30 / 35$ \\
\hline 85.7 & $30 / 35$ \\
\hline 85.3 & $29 / 34$ \\
\hline 87.5 & $28 / 32$ \\
\hline 93.3 & $28 / 30$ \\
\hline 100.0 & $28 / 28$ \\
\hline 100.0 & $28 / 28$ \\
\hline 100.0 & $28 / 28$ \\
\hline 100.0 & $28 / 28$ \\
\hline
\end{tabular}

\begin{tabular}{|c|c|}
\hline \multicolumn{2}{|r|}{ NPV } \\
\hline$\%$ & no./total no. \\
\hline 98.4 & $62 / 63$ \\
\hline 96.9 & $62 / 64$ \\
\hline 96.9 & $62 / 64$ \\
\hline 95.4 & $62 / 65$ \\
\hline 94.0 & $63 / 67$ \\
\hline 94.2 & $65 / 69$ \\
\hline 94.4 & 67/71 \\
\hline 93.1 & $67 / 72$ \\
\hline 88.2 & $67 / 76$ \\
\hline 81.7 & $67 / 82$ \\
\hline
\end{tabular}

NPV, negative predictive value; PAC, plasma aldosterone concentration; PPV, positive predictive value; Sens., Sensitivity; SIT, saline infusion test; Spec., Specificity.

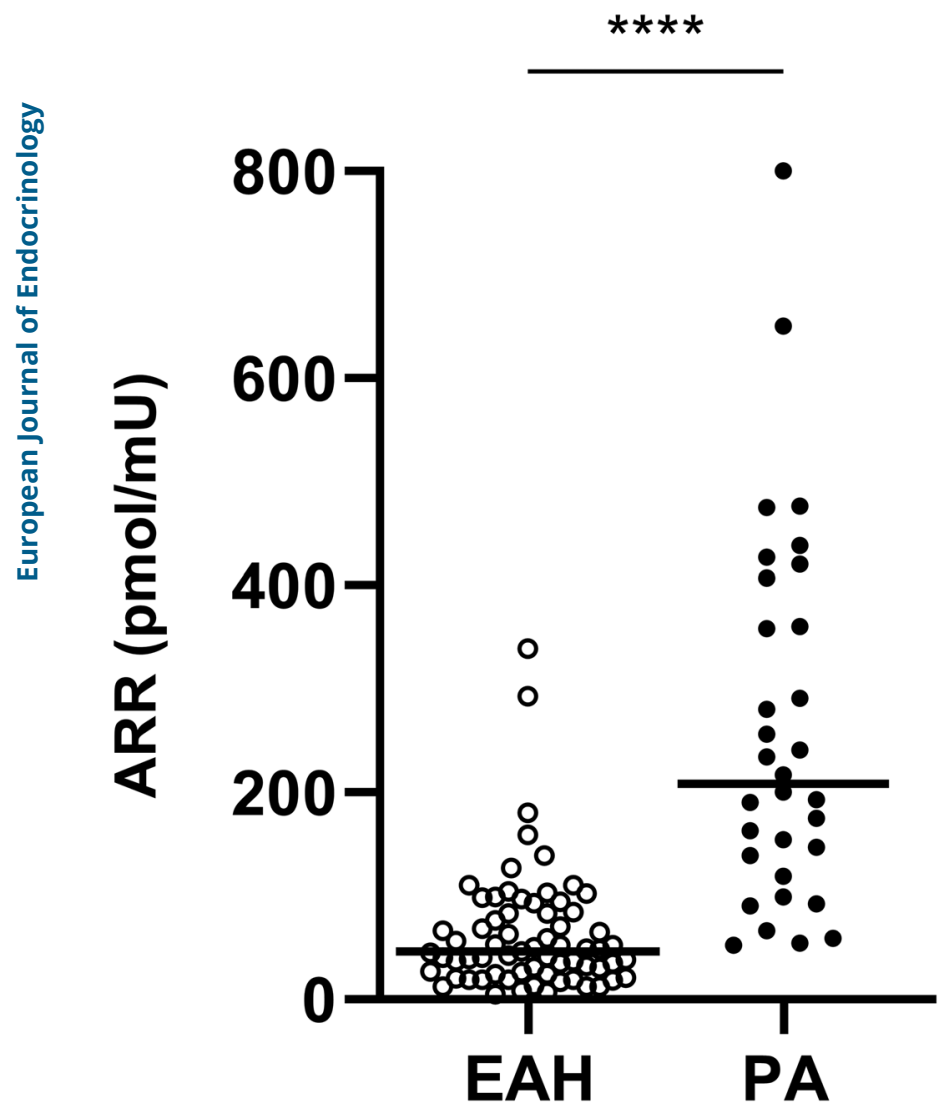

\section{Figure 4}

The diagram shows the aldosterone/renin-ratio (ARR) values in $\mathrm{pmol} / \mathrm{mU}$ of patients diagnosed with primary aldosteronism (PA) and essential arterial hypertension (EAH) obtained at baseline before commencing the saline infusion test (SIT). Median ARR was significantly higher in the PA group compared to $\mathrm{EAH}(P<0.0001)$, although a substantial overlap of value from both groups can be seen. comparability of the most common aldosterone IAs $(23,32,43)$. This is a serious problem for defining a uniform cutoff for PA diagnostic workup and continues to confuse and challenge treating endocrinologists, whose priority is to offer their patients best medical care.

With the development of highly accurate and reproducible LC-MS/MS methods, it became clear that for aldosterone measurement the new gold standard method should be based on LC-MS/MS detection, provided that assay-specific cutoffs are defined (30). The main advantage certainly of LC-MS/MS is its high specificity even at lower PAC levels, showing less inter-laboratory variation and no cross-reactivity with aldosterone metabolites. Moreover, it allows adjusting for specific matrix effects by internal standardization with using stable isotopes $(26,29,44)$. Despite the overall superiority of LC-MS/MS in the aldosterone measurement, this analytical method does not automatically deliver a complete comparability of LC-MS/MS results from different in-house or commercially available approaches. Modifications in the sample pretreatment in the origin of calibrators as well as in the nature of the chromatographic column may still prompt distinct variations in the quantitative readout, which has to be considered (45).

In the present study, we prospectively estimated the diagnostic accuracy of two postulated $\mathrm{PAC}_{\mathrm{LC}-\mathrm{MS} / \mathrm{MS}}$ postinfusion cutoff values for the SIT confirmatory test in an independent cohort of 104 consecutive patients suspected of PA. Overall, our validation cohort revealed a

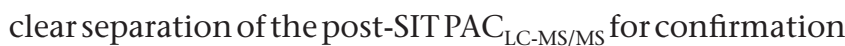
(296.5 pmol/L; IQR 175.8-434.0) vs exclusion of PA (83.0 pmol/L; IQR: 83.0-83.0; Fig. 2A) as well as a significant difference in the median post-SIT PAC between patients sub-classified as IHA (236.0 pmol/L; IQR: 160.0-386.0) and APA (409.0 pmol/L; IQR: 280.0-943.0, Fig. 2B). Since diagnostic uncertainty in PA typically occurs at low PAC, 
Table 5 Screening performance of different ARR pmol/mU values under LC-MS/MS conditions.

\begin{tabular}{|c|c|c|}
\hline \multirow[b]{2}{*}{ ARR $(\mathrm{pmol} / \mathrm{mU})$} & \multicolumn{2}{|c|}{ Sensitivity } \\
\hline & $\%(95 \% \mathrm{Cl})$ & no./total no. \\
\hline$>46$ & $100.0(89.1-100.0)$ & $32 / 32$ \\
\hline$>50$ & $100.0(89.1-100.0)$ & $32 / 32$ \\
\hline$>53$ & 96.9 (83.8-99.9) & $31 / 32$ \\
\hline$>56$ & 93.8 (79.2-99.2) & $30 / 32$ \\
\hline$>60$ & 90.6 (75.0-98.0) & 29/32 \\
\hline$>70$ & 87.5 (71.0-96.5) & $28 / 32$ \\
\hline$>90$ & 84.4 (67.2-94.7) & $27 / 32$ \\
\hline$>110$ & 78.1 (60.0-90.7) & $25 / 32$ \\
\hline
\end{tabular}

\begin{tabular}{|c|c|}
\hline \multicolumn{2}{|c|}{ Specificity } \\
\hline$\%(95 \% \mathrm{Cl})$ & no./total no. \\
\hline 50.8 (38.2-63.2) & $34 / 67$ \\
\hline $55.2(42.6-67.4)$ & $37 / 67$ \\
\hline $59.7(47.0-71.5)$ & $40 / 67$ \\
\hline $61.2(48.5-72.9)$ & $41 / 67$ \\
\hline $62.7(50.0-74.2)$ & $42 / 67$ \\
\hline 70.2 (57.7-80.7) & $47 / 67$ \\
\hline 76.1 (64.1-85.7) & $51 / 67$ \\
\hline $91.0(81.5-96.6)$ & $61 / 67$ \\
\hline
\end{tabular}

\begin{tabular}{|c|c|}
\hline \multicolumn{2}{|r|}{ PPV } \\
\hline$\%$ & no./total no. \\
\hline 49.2 & $32 / 65$ \\
\hline 51.6 & $32 / 62$ \\
\hline 53.4 & $31 / 58$ \\
\hline 53.6 & $30 / 56$ \\
\hline 53.7 & $29 / 54$ \\
\hline 58.3 & $28 / 48$ \\
\hline 62.8 & $27 / 43$ \\
\hline 80.6 & $25 / 31$ \\
\hline
\end{tabular}

\begin{tabular}{|c|c|}
\hline \multicolumn{2}{|c|}{ NPV } \\
\hline$\%$ & no./total no. \\
\hline 100.0 & $34 / 34$ \\
\hline 100.0 & $37 / 37$ \\
\hline 97.6 & $40 / 41$ \\
\hline 95.3 & $41 / 43$ \\
\hline 93.3 & $42 / 45$ \\
\hline 92.2 & $47 / 51$ \\
\hline 91.1 & $51 / 56$ \\
\hline 89.7 & $61 / 68$ \\
\hline
\end{tabular}

ARR, aldosterone/renin-ratio; NPV, negative predictive value; PPV, positive predictive value; Sens., Sensitivity; Spec., Specificity.

where IA-based aldosterone quantification performs most unreliably, the differentiation between IHA and EAH often represents a particular challenge. Even though LC-MS/MS shows markedly lower PAC, the post-SIT $\mathrm{PAC}_{\mathrm{LC}-\mathrm{MS} / \mathrm{MS}}$ offered a good separation even between IHA and EAH (Fig. 2B and Table 3). Overlap of values at low PAC only occurred with ten patients showing postSIT PAC in the diagnostic 'grey area' between 83 and $140 \mathrm{pmol} / \mathrm{L}$. Of these ten patients, five were classified as EAH and five as IHA based on before mentioned diagnostic criteria and CCT results.

Both cutoff values tested here for SIT confirmatory testing performed comparably well, yielding correct diagnosis according to the reference diagnosis in 93 out of 99 patients (93.9\%) using the threshold of $120 \mathrm{pmol} / \mathrm{L}$ and in 92 patients $(92.9 \%)$ using the threshold of $94 \mathrm{pmol} / \mathrm{L}$. As expected, the lower cutoff value deduced more falsely positive $(n=5)$ and less falsely negative diagnoses $(n=2)$ compared to the higher cutoff value, which resulted in over-diagnosing PA in two cases and in missing PA in four cases. Notably, none of the patients who lateralized on AVS were missed with either threshold value, all misclassifications occurred exclusively between the EAH and IHA group.

Because of the importance of a well-balanced diagnostic sensitivity and specificity for confirmatory testing in PA, preventing the patient from unnecessary and possibly harmful consequences of both overdiagnosing and missing PA, we assessed a range of post-

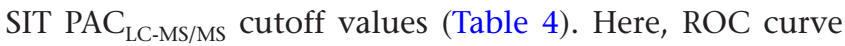
analysis and Youden's index suggest an optimal cutoff value at the LLOQ of $83 \mathrm{pmol} / \mathrm{L}$, where sensitivity is highest at $96.9 \%(95 \%$ CI 83.8-99.9) and specificity at 92.5\% (95\% CI 83.4-97.5). Nevertheless, consequences of a positive confirmatory test in PA are not negligible as invasive procedures such as CT scans, AVS and surgery possibly follow. Placing the focus for confirmatory testing on a higher positive predictive value, an upper post-
SIT threshold value of $130 \mathrm{pmol} / \mathrm{L}$ could be suggested with an indeterminate diagnostic area between 83 and $130 \mathrm{pmol} / \mathrm{L}$ as an equivalent to the IA-derived diagnostic range for post-SIT between 140 and $280 \mathrm{pmol} / \mathrm{L}$ that is commonly used.

Our data confirm that post-SIT cutoff values for diagnosing PA under LC-MS/MS conditions must be considerably lower than established cutoff values under IA conditions to avoid missing PA in a large proportion of patients. In fact, applying the same $\mathrm{PAC}_{\mathrm{IA}}$-derived wide diagnostic range between $<140 \mathrm{pmol} / \mathrm{L}$ (making the diagnosis of PA unlikely) and $>280 \mathrm{pmol} / \mathrm{L}$ (making PA very likely) as suggested by the ES Clinical Practice Guideline (4) to $\mathrm{PAC}_{\mathrm{LC}-\mathrm{MS} / \mathrm{MS}}$ measurement would have resulted in a high number of false negatives: $46.9 \%$ (for post-SIT cutoff of $<280 \mathrm{pmol} / \mathrm{L}$ ), $28.13 \%$ (for cutoff $<190$ $\mathrm{pmol} / \mathrm{L}$ ) and $15.63 \%$ (for cutoff $<140 \mathrm{pmol} / \mathrm{L}$ ). Our results are in line with a recently published study by Stowasser et al. with 100 patients (17) that found a lower PAC of $106 \mathrm{pmol} / \mathrm{L}$ to be most discriminatory as a cutoff value for the recumbent SIT and tested LC-MS/MS assay (26). Taken together, the data presented here support the implication of new method-specific PAC cutoff values under LC-MS/MS conditions.

Finally, for the ARR screening test using $\mathrm{PAC}_{\mathrm{LC}-\mathrm{MS} /}$ MS, Guo et al. (32) recently suggested a ARR screening cutoff of $55 \mathrm{pmol} / \mathrm{mU}$ with a high sensitivity (93.1\%) and reasonable specificity $(87.5 \%)$, which was derived from the comparison results between $\mathrm{PAC}_{\mathrm{IA}}$ and $\mathrm{PAC}_{\mathrm{LS}-\mathrm{MS} / \mathrm{MS}}$ of 41 patients by using regression equation. By contrast, the here suggested $\mathrm{ARR}_{\mathrm{LC}-\mathrm{MS} / \mathrm{MS}}$ screening cutoff of $53 \mathrm{pmol} / \mathrm{mU}$ was derived from ROC curve analysis, thereby resembling the previously calculated optimal $\mathrm{ARR}_{\mathrm{LC}-\mathrm{MS} / \mathrm{MS}}$ screening cutoff value of $52.4 \mathrm{pmol} / \mathrm{mU}$ as reported from Guo et al. (46) and $46 \mathrm{pmol} / \mathrm{mU}$ as reported from Baron et al. (47). This $\mathrm{ARR}_{\mathrm{LC}-\mathrm{MS} / \mathrm{MS}}$ cutoff was chosen as it proved a high sensitivity (96.9\%), a major prerequisite of a screening test, but at the expense of a rather low specificity (60\%). 
The present study has strengths and limitations. The strengths of this study are the prospective validation of pre-specified diagnostic postinfusion threshold values for the SIT confirmatory test, a relatively large sample size of consecutive patients tested under real-life conditions by referral to the university endocrine center for confirmatory testing of PA, and the fact that potentially interfering factors with the PAC and PRC (i.e. circadian timing of testing, intake of ARR-modulating medications, hypokalemia) were controlled and standardized. One critical limitation of the study is the missing diagnostic reference standard for PA. Although the FST may be considered by some authors as a definitive diagnostic procedure (48), it is rather rarely performed in clinical routine as it is comparably time consuming, costly and cumbersome to perform. Moreover, its diagnostic accuracy suffers from the high inter-IAs variability as all other confirmatory tests in PA do. Thus, we feel that a conclusive reference diagnostic approach based on a comprehensive set of clinical, biochemical, functional, morphological and follow-up data secondary to specific therapy is not inferior to a reference diagnosis made by IA-based measurements. Also the choice of the CCT as a second confirmatory test may be criticized given its rather moderate diagnostic accuracy $(10,12,16,49)$. However, the advantage here of the CCT lies in its relative character of tested aldosterone suppression, which appears useful when absolute cutoff values for LC-MS/MS are not available. Another limitation of the presented study is that outcome was not defined according to the Primary Aldosteronism Surgical Outcome (PASO) study criteria. Finally, we do not suggest a general application of the

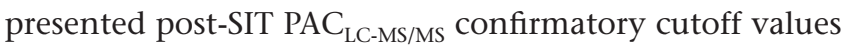
for diagnostic workup of PA. In order to thoughtfully apply the presented values, acceptable method comparability (with $R^{2}>0.90$ ) and validation of the suggested cutoff values at least in a small number of subjects need to be fulfilled (50).

\section{Conclusions}

The present study confirms consistently lower PAC in patients tested for PA under LC-MS/MS conditions and, thus, emphasizes the need for new method-specific diagnostic cutoff values. Proposed $\mathrm{PAC}_{\mathrm{LC}-\mathrm{MS} / \mathrm{MS}}$ cutoff values for the SIT were not inferior to established $\mathrm{PAC}_{\mathrm{IA}}$ values for diagnosing and excluding PA. $\mathrm{PAC}_{\mathrm{LC}-\mathrm{MS} / \mathrm{MS}}$ value of $83 \mathrm{pmol} / \mathrm{L}$ yielded the highest diagnostic accuracy for excluding PA with the recumbent SIT in our study cohort.
A screening ARR value of $53 \mathrm{pmol} / \mathrm{mU}$ was found to be most beneficial.

\section{Declaration of interest}

The authors declare that there is no conflict of interest that could be perceived as prejudicing the impartiality of this study.

\section{Funding}

This research did not receive any specific grant from any funding agency in the public, commercial or not-for-profit sector.

\section{Patient consent}

Consent has been obtained from each patient after full explanation of the purpose and nature of all procedures used.

\section{References}

1 Hannemann A \& Wallaschofski H. Prevalence of primary aldosteronism in patient's cohorts and in population-based studies - a review of the current literature. Hormone and Metabolic Research 201244 157-162. (https://doi.org/10.1055/s-0031-1295438)

2 Monticone S, Burrello J, Tizzani D, Bertello C, Viola A, Buffolo F, Gabetti L, Mengozzi G, Williams TA, Rabbia F et al. Prevalence and clinical manifestations of primary aldosteronism encountered in primary care practice. Journal of the American College of Cardiology 201769 1811-1820. (https://doi.org/10.1016/j.jacc.2017.01.052)

3 Buffolo F, Monticone S, Burrello J, Tetti M, Veglio F, Williams TA \& Mulatero P. Is primary aldosteronism still largely unrecognized?. Hormone and Metabolic Research 201749 908-914. (https://doi. org/10.1055/s-0043-119755)

4 Funder JW, Carey RM, Mantero F, Murad MH, Reincke M, Shibata H, Stowasser M \& Young WF. The management of primary aldosteronism: case detection, diagnosis, and treatment: an Endocrine Society clinical practice guideline. Journal of Clinical Endocrinology and Metabolism 2016101 1889-1916. (https://doi. org/10.1210/jc.2015-4061)

5 Williams TA, Lenders JWM, Mulatero P, Burrello J, Rottenkolber M, Adolf C, Satoh F, Amar L, Quinkler M, Deinum J et al. Outcome of adrenalectomy for unilateral primary aldosteronism: international consensus and remission rates. Lancet: Diabetes and Endocrinology 20175 689-699. (https://doi.org/10.1016/S2213-8587(17)30135-3)

6 Rossi GP, Bernini G, Caliumi C, Desideri G, Fabris B, Ferri C, Ganzaroli C, Giacchetti G, Letizia C, Maccario M et al. A prospective study of the prevalence of primary aldosteronism in 1,125 hypertensive patients. Journal of the American College of Cardiology 200648 2293-2300. (https://doi.org/10.1016/j.jacc.2006.07.059)

7 Stowasser M, Ahmed AH, Pimenta E, Taylor PJ \& Gordon RD. Factors affecting the aldosterone/renin ratio. Hormone and Metabolic Research 201244 170-176. (https://doi.org/10.1055/s-0031-1295460)

8 Tiu SC, Choi CH, Shek CC, Ng YW, Chan FKW, Ng CM \& Kong APS. The use of aldosterone-renin ratio as a diagnostic test for primary hyperaldosteronism and its test characteristics under different conditions of blood sampling. Journal of Clinical Endocrinology and Metabolism 200590 72-78. (https://doi.org/10.1210/jc.2004-1149)

9 Rossi GP, Belfiore A, Bernini G, Desideri G, Fabris B, Ferri C, Giacchetti G, Letizia C, Maccario M, Mallamaci F et al. Comparison of the captopril and the saline infusion test for excluding aldosterone-producing adenoma. Hypertension 200750 424-431. (https://doi.org/10.1161/HYPERTENSIONAHA.107.091827) 
10 Mulatero P, Monticone S, Bertello C, Mengozzi G, Tizzani D, Iannaccone A \& Veglio F. Confirmatory tests in the diagnosis of primary aldosteronism. Hormone and Metabolic Research 201042 406-410. (https://doi.org/10.1055/s-0029-1246186)

11 Nanba K, Tamanaha T, Nakao K, Kawashima ST, Usui T, Tagami T, Okuno H, Shimatsu A, Suzuki T \& Naruse M. Confirmatory testing in primary aldosteronism. Journal of Clinical Endocrinology and Metabolism 201297 1688-1694. (https://doi.org/10.1210/jc.20112504)

12 Song Y, Yang S, He W, Hu J, Cheng Q, Wang Y, Luo T, Ma L, Zhen Q, Zhang S et al. Confirmatory tests for the diagnosis of primary aldosteronism: a prospective diagnostic accuracy study. Hypertension 201871 118-124. (https://doi.org/10.1161/ HYPERTENSIONAHA.117.10197)

13 Rossi GP, Belfiore A, Bernini G, Desideri G, Fabris B, Ferri C, Giacchetti G, Letizia C, Maccario M, Mallamaci F et al. Prospective evaluation of the saline infusion test for excluding primary aldosteronism due to aldosterone-producing adenoma. Journal of Hypertension 200725 1433-1442. (https://doi.org/10.1097/ HJH.0b013e328126856e)

14 Mulatero P, Milan A, Fallo F, Regolisti G, Pizzolo F, Fardella C, Mosso L, Marafetti L, Veglio F \& Maccario M. Comparison of confirmatory tests for the diagnosis of primary aldosteronism. Journal of Clinical Endocrinology and Metabolism 200691 2618-2623. (https:// doi.org/10.1210/jc.2006-0078)

15 Willenberg HS, Vonend O, Schott M, Gao X, Blondin D, Saleh A, Rump LC \& Scherbaum WA. Comparison of the saline infusion test and the fludrocortisone suppression test for the diagnosis of primary aldosteronism. Hormone and Metabolic Research 201244 527-532. (https://doi.org/10.1055/s-0032-1314786)

16 Meng X, Li Y, Wang X, Li J, Liu Y \& Yu Y. Evaluation of the saline infusion test and the captopril challenge test in Chinese patients With primary aldosteronism. Journal of Clinical Endocrinology and Metabolism 2018103 853-860. (https://doi.org/10.1210/jc.201701530)

17 Stowasser M, Ahmed AH, Cowley D, Wolley M, Guo Z, McWhinney BC, Ungerer JP \& Gordon RD. Comparison of seated with recumbent saline suppression testing for the diagnosis of primary aldosteronism. Journal of Clinical Endocrinology and Metabolism 2018103 4113-4124. (https://doi.org/10.1210/jc.2018 01394)

18 Le Goff CM, Gonzalez-Antuña A, Peeters SD, Fabregat-Cabello N, Van Der Gugten JG, Vroonen L, Pottel H, Holmes DT \& Cavalier E. Migration from RIA to LC-MS/MS for aldosterone determination: implications for clinical practice and determination of plasma and urine reference range intervals in a cohort of healthy Belgian subjects. Clinical Mass Spectrometry 20189 7-17. (https://doi. org/10.1016/j.clinms.2018.06.002)

19 Fischer E, Reuschl S, Quinkler M, Rump LC, Hahner S, Bidlingmaier M, Reincke M \& Participants of the German Conn's Registry - Else Kröner-Fresenius-Hyperaldosteronism Registry. Assay characteristics influence the aldosterone to renin ratio as a screening tool for primary aldosteronism: results of the German Conn's registry. Hormone and Metabolic Research 201345 526-531. (https:// doi.org/10.1055/s-0033-1343448)

20 Burrello J, Monticone S, Buffolo F, Lucchiari M, Tetti M, Rabbia F, Mengozzi G, Williams TA, Veglio F \& Mulatero P. Diagnostic accuracy of aldosterone and renin measurement by chemiluminescent immunoassay and radioimmunoassay in primary aldosteronism. Journal of Hypertension 201634 920-927. (https://doi.org/10.1097/ HJH.0000000000000880)

21 Schirpenbach C, Seiler L, Maser-Gluth C, Beuschlein F, Reincke M \& Bidlingmaier M. Automated chemiluminescenceimmunoassay for aldosterone during dynamic testing: comparison to radioimmunoassays with and without extraction steps.
Clinical Chemistry 200652 1749-1755. (https://doi.org/10.1373/ clinchem.2006.068502)

22 Stowasser M, Taylor PJ, Pimenta E, Ahmed AH \& Gordon RD. Laboratory investigation of primary aldosteronism. Clinical Biochemist. Reviews 201031 39-56.

23 Fagotto V, Uhl O, Heinrich D, Nirschl N, Sturm L, Kratzsch J, Bae YJ, Fries C, Fenske W, Reincke M et al. Aldosterone measurement in the diagnosis of primary aldosteronism - comparison between two automated immunoassays and two liquid chromatography tandem mass-spectrometry methods. Endocrine Abstracts 201963 P34. (https://doi.org/10.1530/endoabs.63.P34)

24 Chong YK, Ho CC, Leung SY, Lau SKP \& Woo PCY. Clinical mass spectrometry in the bioinformatics era: a hitchhiker's guide. Computational and Structural Biotechnology Journal 201816 316-334. (https://doi.org/10.1016/j.csbj.2018.08.003)

25 Turpeinen U, Hamalainen E \& Stenman UH. Determination of aldosterone in serum by liquid chromatography-tandem mass spectrometry. Journal of Chromatography: B, Analytical Technologies in the Biomedical and Life Sciences 2008862 113-118. (https://doi. org/10.1016/j.jchromb.2007.11.005)

26 Taylor PJ, Cooper DP, Gordon RD \& Stowasser M. Measurement of aldosterone in human plasma by semiautomated HPLC-tandem mass spectrometry. Clinical Chemistry 200955 1155-1162. (https://doi. org/10.1373/clinchem.2008.116004)

27 Ray JA, Kushnir MM, Palmer J, Sadjadi S, Rockwood AL \& Meikle AW. Enhancement of specificity of aldosterone measurement in human serum and plasma using 2D-LC-MS/MS and comparison with commercial immunoassays. Journal of Chromatography: B, Analytical Technologies in the Biomedical and Life Sciences 2014970 102-107. (https://doi.org/10.1016/j.jchromb.2014.08.042)

28 Gaudl A, Kratzsch J, Bae YJ, Kiess W, Thiery J \& Ceglarek U. Liquid chromatography quadrupole linear ion trap mass spectrometry for quantitative steroid hormone analysis in plasma, urine, saliva and hair. Journal of Chromatography: A 20161464 64-71. (https://doi. org/10.1016/j.chroma.2016.07.087)

29 Van Der Gugten JG, Dubland J, Liu HF, Wang A, Joseph C \& Holmes DT. Determination of serum aldosterone by liquid chromatography and tandem mass spectrometry: a liquid-liquid extraction method for the ABSciex API-5000 mass spectrometry system. Journal of Clinical Pathology 201265 457-462. (https://doi. org/10.1136/jclinpath-2011-200564)

30 Hinchliffe E, Carter S, Owen LJ \& Keevil BG. Quantitation of aldosterone in human plasma by ultra high performance liquid chromatography tandem mass spectrometry. Journal of Chromatography: B, Analytical Technologies in the Biomedical and Life Sciences 2013 913-914 19-23. (https://doi.org/10.1016/j. jchromb.2012.11.013)

31 Juutilainen A, Savolainen K, Romppanen J, Turpeinen U, Hamalainen E, Kemppainen J, Moilanen L \& Pulkki K. Combination of LC-MS/MS aldosterone and automated direct renin in screening for primary aldosteronism. Clinica Chimica Acta: International Journal of Clinical Chemistry 2014433 209-215. (https://doi.org/10.1016/j. cca.2014.03.015)

32 Guo Z, Poglitsch M, McWhinney BC, Ungerer JPJ, Ahmed AH, Gordon RD, Wolley M \& Stowasser M. Aldosterone LC-MS/MS assay-specific threshold values in screening and confirmatory testing for primary aldosteronism. Journal of Clinical Endocrinology and Metabolism 2018103 3965-3973. (https://doi.org/10.1210/jc.201801041)

33 Bae YJ \& Kratzsch J. Data from: Comparison of aldosterone LC-MS/ MS assay vs. immunoassay: Figshare 2016. Deposited 17.10.2019, https://doi.org/10.6084/m9.figshare.9949046.v1.

34 Giacchetti G, Ronconi V, Lucarelli G, Boscaro M \& Mantero F. Analysis of screening and confirmatory tests in the diagnosis of primary aldosteronism: need for a standardized protocol. Journal 
of Hypertension 200624 737-745. (https://doi.org/10.1097/01. hjh.0000217857.20241.0f)

35 Holland OB, Brown H, Kuhnert L, Fairchild C, Risk M \& GomezSanchez CE. Further evaluation of saline infusion for the diagnosis of primary aldosteronism. Hypertension 19846 717-723. (https://doi. org/10.1161/01.HYP.6.5.717)

36 Kem DC, Weinberger MH, Mayes DM \& Nugent CA. Saline suppression of plasma aldosterone in hypertension. Archives of Internal Medicine 1971128 380-386. (https://doi.org/10.1001/archi nte.1971.00310210056004)

37 Gerards J, Heinrich DA, Adolf C, Meisinger C, Rathmann W, Sturm L, Nirschl N, Bidlingmaier M, Beuschlein F, Thorand B et al. Impaired glucose metabolism in primary aldosteronism is associated With cortisol co-secretion. Journal of Clinical Endocrinology and Metabolism 2019104 3192-3202. (https://doi.org/10.1210/jc.2019-00299)

38 Reincke M, Fischer E, Gerum S, Merkle K, Schulz S, Pallauf A, Quinkler M, Hanslik G, Lang K, Hahner S et al. Observational study mortality in treated primary aldosteronism: the German Conn's registry. Hypertension 201260 618-624. (https://doi.org/10.1161/ HYPERTENSIONAHA.112.197111)

39 Lyons DF, Kem DC, Brown RD, Hanson CS \& Carollo ML. Single dose captopril as a diagnostic test for primary aldosteronism. Journal of Clinical Endocrinology and Metabolism 198357 892-896. (https://doi. org/10.1210/jcem-57-5-892)

40 Fuss CT, Treitl M, Rayes N, Podrabsky P, Fenske WK, Heinrich DA, Reincke M, Petersen TO, Fassnacht M, Quinkler M et al. Radiation exposure of adrenal vein sampling: a German Multicenter Study. European Journal of Endocrinology 2018179 261-267. (https://doi. org/10.1530/EJE-18-0328)

41 Ahmed AH, Gordon RD, Taylor PJ, Ward G, Pimenta E \& Stowasser M. Are women more at risk of false-positive primary aldosteronism screening and unnecessary suppression testing than men? Journal of Clinical Endocrinology and Metabolism 201196 E340-E346. (https://doi.org/10.1210/jc.2010-1355)

42 Schirpenbach C, Segmiller F, Diederich S, Hahner S, Lorenz R, Rump LC, Seufert J, Quinkler M, Bidlingmaier M, Beuschlein F et al. The diagnosis and treatment of primary hyperaldosteronism in Germany: results on 555 patients from the German Conn Registry. Deutsches Arzteblatt international 2009106 305-311. (https://doi. org/10.3238/arztebl.2009.0305)
43 Mulatero P, Dluhy RG, Giacchetti G, Boscaro M, Veglio F \& Stewart PM. Diagnosis of primary aldosteronism: from screening to subtype differentiation. Trends in Endocrinology and Metabolism 2005 16 114-119. (https://doi.org/10.1016/j.tem.2005.02.007)

44 Kushnir MM, Rockwood AL \& Bergquist J. Liquid chromatographytandem mass spectrometry applications in endocrinology. Mass Spectrometry Reviews 201029 480-502. (https://doi.org/10.1002/ mas.20264)

45 Nishikawa T, Omura M, Kawaguchi M, Takatsu A, Satoh F, Ito S, Kurihara I, Itoh H, Yanase T, Shibata $\mathrm{H}$ et al. Calibration and evaluation of routine methods by serum certified reference material for aldosterone measurement in blood Endocrine Journal 201663 1065-1080. (https://doi.org/10.1507/ endocrj.EJ16-0304)

46 Guo Z, Poglitsch M, Mcwhinney B, Ungerer J, Ahmed A, Gordon R, Wolley M, Stowasser M, Guo Z, Poglitsch M et al. Comparison between the aldosterone/renin ratio and the aldosterone/angiotensin II ratio as a screening test for primary aldosteronism. In Annual Scientific Meeting of the High Blood Pressure Research Council of Australia, Melbourne, Australia, Abstract 15, 2017.

47 Baron S, Amar L, Faucon AL, Blanchard A, Baffalie L, Faucard C, Travers S, Pagny JY, Azizi M \& Houillier P. Criteria for diagnosing primary aldosteronism on the basis of liquid chromatographytandem mass spectrometry determinations of plasma aldosterone concentration. Journal of Hypertension 201836 1592-1601. (https:// doi.org/10.1097/HJH.0000000000001735)

48 Stowasser M \& Gordon RD. Primary aldosteronism. Best Practice and Research Clinical Endocrinology and Metabolism 200317 591-605. (https://doi.org/10.1016/S1521-690X(03)00050-2)

49 Mulatero P, Bertello C, Garrone C, Rossato D, Mengozzi G, Verhovez A, Fallo F \& Veglio F. Captopril test can give misleading results in patients with suspect primary aldosteronism. Hypertension 200750 e26-e27. (https://doi.org/10.1161/ HYPERTENSIONAHA.107.093468)

50 Bae YJ, Zeidler R, Baber R, Vogel M, Wirkner K, Loeffler M, Ceglarek U, Kiess W, Körner A, Thiery J et al. Reference intervals of nine steroid hormones over the life-span analyzed by LC-MS/MS: effect of age, gender, puberty, and oral contraceptives. Journal of Steroid Biochemistry and Molecular Biology 2019193 105409. (https:// doi.org/10.1016/j.jsbmb.2019.105409)

Received 13 January 2020

Revised version received 27 April 2020

Accepted 26 May 2020 